\title{
Ecología y bioética
}

\section{Un futuro por construir: la propuesta del ambientalismo moderno}

A future to build: the proposal of modern environmentalism

B) Autor

\author{
Esteban Rossi \\ Instituto de Bioética, Pontificia Universidad Javeriana \\ E-mail: esteban.rossi@javeriana.edu.co
}




\section{Resumen}

Abstract

Key words

Ecomodernismo; energía; cambio climático; desacoplamiento; desarrollo.

Ecomodernism; energy; climate change; decoupling; development.

En las últimas dos décadas el movimiento ambientalista ha experimentado cambios profundos que han dado origen a nuevos discursos. En conjunto, estos discursos han tomado el hombre de ambientalismo moderno 0 ecomodernismo. Este trabajo examina las principales propuestas del ambientalismo moderno con énfasis en la relación entre el ambiente, la energía y el desarrollo. Se describen también las ideas ético-políticas en que se apoya este discurso y que le permiten articular una visión optimista sobre el futuro de la humanidad. Se observa que el enfoque pragmático e interdisciplinario del ambientalismo moderno permite proponer soluciones prácticas a los problemas socio-ambientales, construir acuerdos entre actores muy distintos, y establecer puentes entre áreas del conocimiento. El ambientalismo moderno ofrece también una novedosa interpretación del proceso de desarrollo ligada a una ambiciosa apuesta política. En conclusión, las propuestas del ambientalismo moderno probablemente merecen atenta consideración.

In the last two decades the environmental movement has experienced deep changes that have generated new discourses. As a whole, said discourses have adopted the name of modern environmentalism or ecomodernism. This article analyses the main proposals of modern environmentalism focusing on the relation between environment, energy and development. It also describes the ethical-political ideas on which this discourse is based and which allow to create an optimistic view about the future of humanity. It is noted that the pragmatic and interdisciplinary approach of modern environmentalism allows suggesting practical solutions for the social and environmental problems, building agreements among very different actors and bridges between knowledge areas. Modern environmentalism also offers a new interpretation of the development process linked to an ambitious political proposal. In conclusion, modern environmentalism proposals probably deserve careful consideration. 


\section{Introducción}

\subsection{El ambientalismo}

En los países occidentales el movimiento ambientalista se origina como una reacción a los efectos negativos de la industrialización luego de la segunda guerra mundial, particularmente en la década de 1960. Los trabajos de John Muir, Aldo Leopold y Rachel Carlson permitieron comunicar las cambiantes concepciones del ambiente y sus implicaciones sociales en Norteamérica y posteriormente en otros países ${ }^{1}$. Desde entonces diversos grupos sociales han rechazado el uso inadecuado de pesticidas y fertilizantes, la transformación del paisaje, la pérdi-

\section{El creciente interés por los}

temas ambientales ha generado

un profundo cambio cultural que

se observa en las instituciones

educativas, la empresa privada

y los gobiernos da de biodiversidad y la contaminación del aire y el agua por el uso de combustibles fósiles. Eventualmente, estas reacciones confluyeron en un movimiento enfocado en la protección del ambiente y en la reivindicación de los derechos de las personas a disfrutar de un ambiente sano².

De igual manera, la consolidación del movimiento ambientalista se debe también a la creciente prosperidad material que tuvo lugar entre 1940 y 1970 . Aunque los efectos negativos de la industrialización fueron notorios desde principios del siglo XX, solo recibieron atención a partir de los años sesenta cuando la mayor parte de la población había alcanzado un nivel de prosperidad material considerable. El crecimiento económico de la posguerra junto con la inversión estatal en infraestructura y programas sociales permitieron que Estados Unidos y posteriormente Europa Occidental alcanzaran niveles de bienestar sin precedentes ${ }^{3}$. En Estados Unidos la creciente afluencia durante los años sesenta y setenta facilitó la aprobación de leyes para la protección del aire, el agua y la biodiversidad, que mejoraron la calidad del ambiente y continúan vigentes. Paralelamente, la comunidad internacional estableció un foro dedicado a temas ambientales y señaló la importancia de la reducción de la capa de ozono, la deforestación tropical y el cambio climático ${ }^{4}$. El creciente interés por los temas ambientales ha generado un profundo cambio cultural que se observa en las instituciones educativas, la empresa privada y los gobiernos. De igual manera, el uso eficiente del agua, el consumo responsable y el reciclaje forman parte de los procesos de numerosas instituciones alrededor del mundo. En resumen, gracias a los esfuerzos de los ambientalistas de generaciones anteriores, el movimiento ambientalista ha impulsado valiosos cambios culturales y políticos.

1 Kareiva, P., Marvier, M., Lalasz, R. (2012). Conservation in the Anthropocene. Breakthrough Journal. Winter. http://thebreakthrough.org/ index.php/journal/past-issues/issue-2/conservation-in-the-anthropocene.

2 Kareiva, P. (2011). Failed Metaphors and a New Environmentalism for the 21st Century. https://www.youtube.com/ watch?v=4BOEQkvCook.

3 Nordhaus, T. and Shellenberger, M. (2007). BREAK-THROUGH. New York: Houghton Miflin, 344.

4 Speth, J. G. (2005). Red Sky at Morning: America and the Crisis of the Global Environment. Yale University Press, 328. 
Sin embargo, sin demeritar estos logros, el movimiento ambientalista contemporáneo presenta problemas que requieren atención. Primero, el ambientalismo no ha reflejado las necesidades de los países más pobres, por el contrario entra frecuentemente en conflicto con ellas. Segundo, numerosas propuestas ambientalistas buscan definir límites estrictos para el uso de los recursos y el crecimiento económico. Esta visión también choca con diversos proyectos políticos que dependen del crecimiento económico para eliminar la pobreza y que reclaman cambios fundamentales en las relaciones sociales.

El movimiento ambientalista contemporáneo presenta problemas que requieren atención. Primero, el ambientalismo no ha reflejado las necesidades de los países más pobres, por el contrario entra frecuentemente en conflicto con ellas En tercer lugar, la distribución de los costos de la protección del ambiente genera problemas éticos que el ambientalismo tradicional no ha podido resolver. Este trabajo describe la forma en que el ambientalismo moderno aborda estas limitaciones del ambientalismo tradicional. Se utiliza un enfoque que combina información científica y técnica junto con apreciaciones teóricas de tipo humanístico. Este enfoque interdisciplinario busca establecer oportunidades de discusión tanto con científicos interesados en caracterizar procesos ecológicos, como con académicos y activistas interesados en fenómenos de tipo social o político. El objetivo de este trabajo consiste en señalar cómo las propuestas del ambientalismo moderno podrían permitir enfrentar algunos de los problemas más complejos del presente, específicamente la necesidad de erradicar definitivamente la pobreza material y la contaminación derivada del uso de combustibles fósiles. En la primera sección se describe cómo la tensión entre la eliminación de la pobreza y la preservación del ambiente impulsa la búsqueda de una nueva visión. La segunda sección describe el papel de la energía junto con la importancia del proceso de cambio tecnológico. Por último, la tercera sección explora las ideas políticas del ambientalismo moderno, particularmente su visión optimista de un futuro próspero para la humanidad y otras especies.

\subsection{Tensión entre el desarrollo y el ambiente}

En su concepción más simple el desarrollo socioeconómico consiste en utilizar recursos naturales como el agua, la madera o el suelo para satisfacer necesidades humanas y causa transformaciones importantes en los ecosistemas. Aunque el desarrollo se puede medir de varias maneras, de forma simplificada como producto interno bruto (PIB), o de forma más integral como el índice de desarrollo humano de las Naciones Unidas, por lo general, se reconoce que el desarrollo demanda el uso de recursos ${ }^{5}$. La agricultura y la urbanización, por ejemplo, generan importantes beneficios para las personas y causan cambios en los ecosistemas. De forma similar, los combustibles fósiles alimentan la economía mundial, pero su uso causa contaminación del aire y el agua. Existen dos estrategias principales para abordar esta tensión: limitar el uso de recursos buscando reducir la polución, o acelerar el proceso de desarrollo para hacerlo menos

5 United Nations Development Program (ND). Datos sobre el desarrollo humano (1980-2015). United Nations development programme. Recuperado de: http://hdr.undp.org/es/data. 
contaminante posteriormente. Históricamente, el movimiento ambientalista ha estado dominado por la primera estrategia, es decir ha buscado establecer límites al uso de recursos. Por ejemplo, desde hace casi dos décadas, la comunidad ambientalista ha insistido, sin éxito, en restringir el uso de combustibles fósiles para reducir las emisiones de carbono. En respuesta, numerosos líderes de países en desarrollo han señalado que su prioridad consiste en erradicar la pobreza para lo cual requieren de combustibles fósiles. Debido a su abundancia, accesibilidad y costo, los combustibles fósiles constituyen la principal fuente de energía de la economía mundial ${ }^{6}$. De esta forma las prescripciones de los ambientalistas chocan con los intereses de los países en desarrollo.

Esta tensión se ha observado repetidamente en los procesos internacionales de la cumbre del clima. Brasil, India y China, actores importantes de la economía mundial, se han negado a limitar su crecimiento económico para reducir sus emisiones de carbono. Debido a estos desacuerdos, el Protocolo de Kioto de 1997, la cumbre de Copenhague en 2009, e incluso el Acuerdo de París en 2016, han tenido resultados pobres ${ }^{7}$. Paralelamente las emisiones globales de carbono se han duplicado desde $1990^{8}$ (figura 1). Sin embargo, vale la pena resaltar que desde 2015 las emisiones globales han aumentado a una tasa del $1 \%$ aproximadamente mientras que el PIB global ha sido del $3 \%$ sugiriendo que el crecimiento económico no depende completamente de los combustibles fósiles.

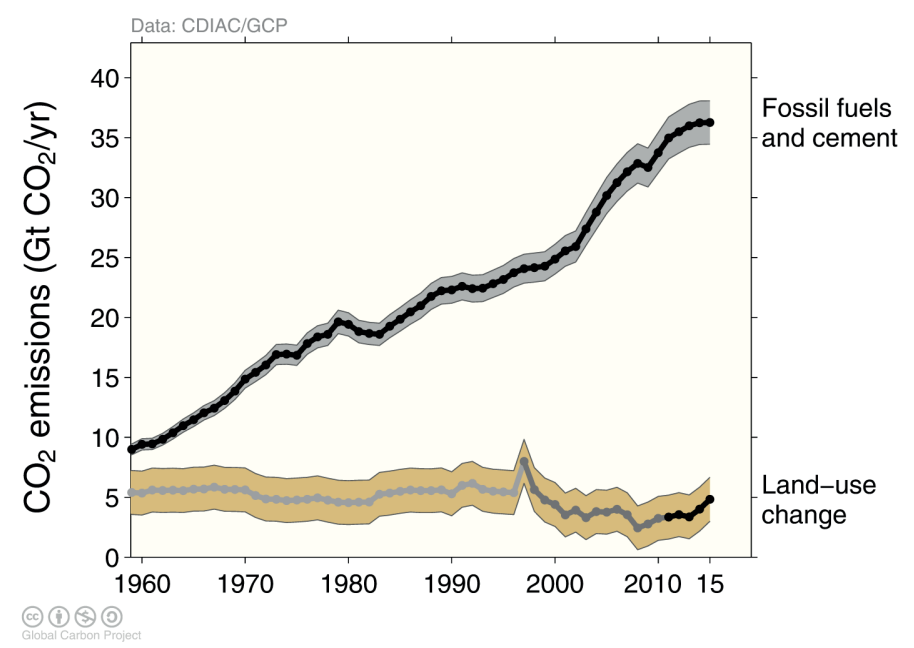

Figura 1. Emisiones totales de carbón en giga toneladas.

6 Lula da Silva, L. I. (2009). Speech of the Brazilian President Lula at the Conference of the Parties, in Copehagen (COP15), Part 1. [Video file] https://www.youtube.com/watch?v=LQzVjDp5WA8.

7 Lomborg, B. (2016). Impact of Current Climate Proposals. [Versión digital] Glob Policy, 7 109-118. Recuperado de: https://doi. org/10.1111/1758-5899.12295.

Lomborg, B. (2016). The Paris agreement was never the solution to climate change. https://capx.co/paris-was-never-the-solution/ [Video file] https://youtu.be/47bNzLj5E_Q.

8 Le Quéré, C. et al. (2016). Global Carbon Budget 2016. [Versión digital] Earth Syst. Sci. Data, 8, 605-649. Recuperado de: https://doi. org/10.5194/essd-8-605-2016. http://www.earth-syst-sci-data.net/8/605/2016/.

Website: http://www.globalcarbonproject.org/carbonbudget/16/highlights.htm. 
En el año 2015 se emitieron $41.9 \pm 2.8$ GtCO2 lo que equivale a un aumento de $49 \%$ con respecto a 1990. Porcentaje de emisiones provenientes de cambios en el uso de la tierra fue de 9-10\% para el periodo 2006-2015. Datos de CDIAC; Houghton et al., 2012; Giglio et al., 2013; Le Quéré et al., 2016; Global Carbon Budget, 2016.

Adicionalmente, la crisis económica de 2008-2009 sumada al pesimismo de algunos grupos ambientalistas han causado fatiga y apatía entre el público $0^{9}$. Algunos sondeos muestran que el medioambiente y el cambio climático no son prioridades para los ciudadanos de países desarrollados ni tampoco para los ciudada-

Desde los años noventa el

movimiento ambientalista

ha sido incapaz de articular

una propuesta que permita

formar coaliciones entre

países en desarrollo y países

industrializados. Por tanto, se

requiere una nueva visión que

refleje los problemas políticos

y económicos del presente y

que permita buscar soluciones

concertadas nos de países pobres ${ }^{10}$. Ante problemas como el desempleo o la inestabilidad económica, el medioambiente ha perdido protagonismo en la esfera pública ${ }^{11}$. En parte por esta razón proliferan iniciativas comerciales del llamado "capitalismo cultural" que buscan enlazar los actos de consumo individual con la protección del ambiente. Entre estas iniciativas se encuentran el café orgánico de comercio justo, el chocolate socialmente responsable y diversos productos producidos por cooperativas y que buscan llegar al consumidor sin la mediación de intermediarios $^{12}$. Las debilidades de estos esfuerzos por individualizar la protección del medioambiente saltan a la vista: la suma de actos de consumo individuales no es suficiente para abordar los problemas ambientales ${ }^{13}$. El uso de bombillas led, el ahorro de agua en las escuelas y el uso de la bicicleta tienen un inmenso valor educativo y local, pero son insignificantes desde el punto de vista global ${ }^{14}$. Por ejemplo, sustituir diez bombillas incandescentes por bombillas led puede ahorrar 0.68 toneladas de $\mathrm{CO}_{2}$ al año en un hogar, luego si cinco millones de hogares reemplazan diez bombillas cada uno, la reducción total será de 3.4 millones de toneladas $\mathrm{CO}_{2}$. Esta cifra parece significativa, pero si consideramos que en 2015 las emisiones de España superaron las 200 millones de toneladas de $\mathrm{CO}_{2}$ (300 millones si se incluyen gases como el metano), es claro que los esfuerzos individuales son insignificantes para reducir la contaminación. Los esfuerzos individuales se encuentran órdenes de magnitud por debajo de lo que se requiere para estabilizar la concentración de $\mathrm{CO}_{2}$ en la atmosfera. Por tanto es necesario desarrollar estrategias colectivas para

9 Nordhaus, T. and Shellenberger, M. (2009). Apocalypse Fatigue: Losing the Public on Climate Change. http://e360.yale.edu/features/ apocalypse_fatigue_losing_the_public_on_climate_change.

10 PEW research center, (2009). Fewer Americans See Solid Evidence of Global Warming. http://www.people-press.org/2009/10/22/ fewer-americans-see-solid-evidence-of-global-warming/.

11 Swyngedouw, E. (2010). Apocalypse Forever? [Versión digital] Theory, Culture \& Society 27 (2-3) 213-232. Recuperado de: https://doi. org/10.1177/0263276409358728.

12 Zizek, S. (2011). Cultural Capitalism. [Video file] https://www.youtube.com/watch?v=GRvRm19UKdA.

13 Maniates, M. F. (2001). Individualization: Plant a Tree; Buy a Bike, Save the World? [Versión digital] Global Environmental Politics 1 (3) 31-52. Recuperado de: https://doi.org/10.1162/152638001316881395.

14 Tierney, J. (2007). 'Feel Good' vs. 'Do Good' on Climate. The New York Times. http://www.nytimes.com/2007/09/11/science/ earth/11tiern.html. 
reducir las emisiones. En resumen, desde los años noventa el movimiento ambientalista ha sido incapaz de articular una propuesta que permita formar coaliciones entre países en desarrollo y países industrializados. Por tanto, se requiere una nueva visión que refleje los problemas políticos y económicos del presente y que permita buscar soluciones concertadas.

El ambientalismo moderno señala que el bienestar de los ecosistemas y las personas son inseparables, por esta razón para resolver algunos problemas ambientales es necesario eliminar la pobreza material. Debido a que la erradicación de la miseria requiere ampliar radicalmente el acceso a la energía de las personas más pobres alrededor del mundo, es importante reconocer que aumentar el uso de la energía generará algunos impactos ambientales. De igual manera, dado que el desarrollo y los problemas ambientales se encuentran ligados, el ambientalismo moderno propone redefinir el problema del cambio climático. El fenómeno del cambio climático se debe conceptualizar como un problema de inadecuado o insuficiente desarrollo, en vez de como un problema de exceso de polución ${ }^{15}$. A continuación se describe el papel de la energía en la propuesta ecomodernista.

\section{Nuevo ambientalismo}

\subsection{Energía}

Durante la próxima década la mayor parte del crecimiento

de la matriz energética global

tendrá lugar en áreas urbanas

en países en desarrollo
El acceso a la energía a un bajo costo es fundamental para el desarrollo. El estilo de vida moderno depende de grandes cantidades de energía. Basta mencionar el aire acondicionado y la calefacción, los computadores y los teléfonos móviles, y los sistemas de refrigeración para alimentos y medicamentos, estos servicios dependen de la disponibilidad permanente de energía. Desafortunadamente, miles de millones de personas distribuidas alrededor del mundo todavía no cuentan con acceso a electricidad y casi tres mil millones cocinan con leña, estiércol o carbón. Para estas personas el acceso limitado a la energía reduce notablemente su calidad de vida y constituye un riesgo para su salud. Cocinar con leña o carbón en espacios cerrados causa enfermedades pulmonares en más de un millón de personas a nivel mundial, principalmente mujeres ${ }^{16}$. Por consiguiente, erradicar la pobreza demandará mucha energía en las próximas décadas. Debido a su importancia para el desarrollo, el ambientalismo moderno propone definir el acceso a la energía como un bien público y ampliar significativamente su disponibilidad entre los más pobres.

15 Asafu-Adjaye, J. et al. (2015). El Manifesto Ecomodernista. http://www.ecomodernism.org/espanol/.

16 Fullerton, D. G., Bruce, N., Gordon, S. B. (2008). Indoor air pollution from biomass fuel smoke is a major health concern in the developing world. [Versión digital] Trans R Soc Trop Med Hyg, 102 (9) 843-851. Recuperado de: https://doi.org/10.1016/j.trstmh.2008.05.028. 
Durante la próxima década la mayor parte del crecimiento de la matriz energética global tendrá lugar en áreas urbanas en países en desarrollo. Por lo tanto su expansión y mejoramiento dependerá de inversiones públicas y privadas. La adecuada articulación entre los actores permitirá generar economías de escala que reduzcan los costos y garanticen que la totalidad de la población tenga acceso a energía eléctrica. Es importante resaltar que mientras Estados Unidos e Inglaterra lograron completa cobertura de la matriz energética en la década de 1960, en India el 20\% de la población todavía no tiene acceso a electricidad y en África más del $50 \%$ de la población no está conectada a la matriz ${ }^{17}$.

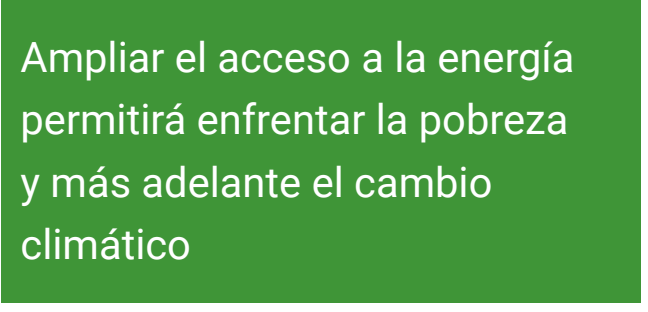

El ambientalismo moderno señala que la consolidación de una matriz eléctrica facilitará la transición hacia fuentes de energía más limpias. Esa fue la trayectoria de los países industrializados desde la primera revolución industrial. El primer combustible industrial fue el carbón, seguido del petróleo, y más recientemente el gas natural. En el futuro, esta transición ocurrirá más rápidamente, por lo cual algunos expertos aseguran que con inversión y estímulos adecuados el gas natural podría reemplazar al petróleo en casi todos sus usos para convertirse en el combustible del siglo $\mathrm{XXI}^{18}$. El gas natural es menos contaminante que el petróleo y produce la mitad del $\mathrm{CO}_{2}$ que el carbón.

Posteriormente, en una matriz rentable y en expansión, la energía nuclear podría reemplazar al gas, para luego complementarse con energía renovable si la tecnología está disponible en ese momento. De esta forma, en el transcurso de aproximadamente tres o cuatro décadas se podría garantizar el acceso a electricidad para la totalidad de la población mundial y establecer las bases para una economía sin emisiones de carbono. Aunque existen numerosas incertidumbres, el análisis de las pasadas transiciones energéticas arroja tres conclusiones. Primero, el proceso de transición es lento y requiere la coordinación entre actores privados y públicos. Construir redes de transmisión eléctricas es costoso por lo que las políticas públicas pueden acelerar o retrasar el proceso. Segundo, las economías de escala facilitan la transición hacia fuentes más limpias y favorecen la innovación. Tercero, experiencias recientes con la perforación horizontal y la fracturación hidráulica muestran que el avance tecnológico aumentó la disponibilidad y redujo los costos del gas natural. En conjunto, estos avances favorecen la transición del petróleo hacia el gas. En resumen, ampliar el acceso a la energía permitirá enfrentar la pobreza y más adelante el cambio climático. El papel de la tecnología en estos procesos se describe en la siguiente sección.

17 World Bank. Access to electricity (\% of population) http://data.worldbank.org/indicator.

Lindeman, T. (2012). 1.3 billion are living in the dark. The Washington Post. https://www.washingtonpost.com/graphics/world/worldwithout-power/.

Dolla, C. N. H., Pachauric, S. (2010). Estimating rural populations without access to electricity in developing countries through nighttime light satellite imagery. Energy Policy, 38 (10) 5661-5670.

18 Smil, V. (2015). Natural Gas: Fuel for the 21st Century. Wiley, p. 264. 


\subsection{Cambio tecnológico}

El cambio tecnológico es un proceso complejo que abarca un conjunto de disciplinas del conocimiento y se puede abordar a distintas escalas. Este análisis se enfoca en tres aspectos de este proceso que tienen claras implicaciones ambientales: (1) el desacoplamiento (decoupling) de la economía y el impacto ambiental, (2) la gradual descarbonización de la economía y (3) los avances en las

En Estados Unidos, los patrones
de consumo se han venido esta-
bilizando y en algunos casos
reduciendo desde 1970 . Por
ejemplo, el área dedicada a la
agricultura, particularmente el
maíz y patatas se ha mantenido
constante
tecnologías de la información. El concepto de desacoplamiento se refiere a la relación entre el crecimiento económico, como producto interno bruto (PIB) y la cantidad de energía y materiales utilizados $^{19}$. Datos de diversas fuentes indican que mientras el PIB aumenta a una tasa de aproximadamente $3 \%$ cada año, el uso de energía y materiales aumenta a una tasa mucho menor. En Estados Unidos se estima que desde 1800 la cantidad de energía requerida para fabricar un producto cualquiera es un $1 \%$ menor que el año anterior. Así el PIB está separándose gradual y parcialmente del impacto ambiental. Las reducciones en el impacto ambiental se atribuyen a un aumento en la eficiencia en la producción y cambios en los patrones de uso de los recursos.

En Estados Unidos, los patrones de consumo se han venido estabilizando y en algunos casos reduciendo desde 1970. Por ejemplo, el área dedicada a la agricultura, particularmente el maíz y las patatas se ha mantenido constante, mientras que la producción ha aumentado gradualmente. Globalmente, el número de hectáreas agrícolas se estabilizó a partir de $2010^{20}$. De forma similar, en países como Francia y Escocia el área forestal viene aumentando desde hace un siglo. La expansión forestal se encuentra asociada al abandono de la agricultura de subsistencia y a la urbanización y se denomina transición forestal ${ }^{21}$. Se calcula que en Estados Unidos la transición forestal se completó alrededor del año $1990^{22}$.

Por otra parte, el uso de algunos minerales también ha descendido gradualmente en las últimas dos décadas. En Estados Unidos, el uso de cobre, acero y aluminio entre otros, se ha reducido desde el año 2000 , lo que indica que el consumo de materiales no aumenta a la misma tasa a la que crece la economía. En conjunto, las observaciones de las últimas décadas sugieren que hay un desacoplamiento relativo entre el PIB y el uso de materiales. Esto no significa que el impacto ambiental absoluto se reduzca, o que el consumo no genere problemas locales. Sin embargo, estas tendencias nos invitan a revisar algunos supuestos del discurso ambientalista tradicional. Algunos de estos

19 Ward, J. D., et al. (2016). Is Decoupling GDP Growth from Environmental Impact Possible? [Versión digital] PLoS ONE, 11 (10) e0164733. Recuperado de: https://doi.org/10.1371/journal.pone.0164733.

20 Ausubel, J. H., Wernick, I. K. and Waggoner, P. E. (2013). Peak Farmland and the Prospect for Land Sparing. [Versión digital] Population and Development Review, 38, 221-242. Recuperado de: https://doi.org/10.1111/j.1728-4457.2013.00561.x

21 Mather, A. S. and Needle, C. L. (1998). The forest transition: a theoretical basis. [Versión digital] Area, 30, 117-124. Recuperado de: https://doi.org/10.1111/j.1475-4762.1998.tb00055.x

22 Cronon, W. (1983). Changes in the Land: Indians, Colonists, and the Ecology of New England. New York: Hill and Wang. 
supuestos fueron establecidos en los años setenta cuando la tasa de crecimiento de la población estaba en el punto más alto del siglo XX. Sin embargo, datos recopilados en las últimas tres décadas señalan que no hay escasez global de recursos.

La descarbonización es el proceso de reducción de la cantidad de carbono emitida por unidad de energía. Existen varias estrategias para reducir la cantidad de carbono proveniente de la utilización de combustibles fósiles. Primero, la reducción en las emisiones mediante aumentos en la eficiencia, el ahorro de energía y el uso de combustibles fósiles más densos en energía. Segundo, la captura y almacenamiento de carbono (CAC) por diversos mecanismos. Y tercero, la eliminación del carbono mediante el uso de fuentes de energía limpias. Las fuentes de energía que no liberan carbono incluyen las plantas nucleares, las renovables (hidroeléctrica, solar y eóli-

En China más del $70 \%$ de

la energía proviene de la

combustión del carbón. Por

consiguiente, si se busca

estabilizar las emisiones en

el corto plazo será necesario

ampliar la inversión en captura y

almacenamiento del carbono ca), los biocombustibles y el hidrógeno. Por razones de espacio, a continuación se describe el primer mecanismo y se resumen brevemente el segundo y el tercero. En los últimos 200 años la tasa global de descarbonización ha sido del $0.3 \%$ por año, este lento proceso ha permitido que desde 1860 el número de toneladas de carbono emitidas se reduzca un $40 \%$. Desde el punto de vista económico la descarbonización se observa como la decreciente cantidad de carbono requerida para producir un dólar ajustado de PIB. En Estados Unidos, se estima que mientras en 1800 se requerían tres kilos de carbono para producir un dólar de PIB, en 1990 se requerían solamente 0.3 kilos. Estos graduales pero notables avances en la eficiencia se deben en parte a la expansión de una economía basada en servicios y la automatización de la industria manufacturera. Sin embargo, vale la pena resaltar que en la actualidad la intensidad de carbono por unidad de PIB en India y China se asemeja a la de Estados Unidos e Inglaterra durante el siglo XIX ${ }^{23}$ por lo que la descarbonización de la economía global será un proceso de larga duración. Sin embargo, al parecer estamos cerca del pico global de emisiones y es posible que la descarbonización se acelere en los próximos años.

La captura y almacenamiento de carbono (CAC) busca recoger y almacenar el $\mathrm{CO}_{2}$ después de la combustión antes que sea liberado a la atmósfera. Para algunos la CAC es un elemento importante del portafolio de opciones de mitigación debido a la importancia actual de los combustibles fósiles. La CAC es popular entre productores y consumidores de carbón que esperan modificar sus plantas generadoras para capturar las emisiones en la fuente y continuar utilizando carbón. En China más del 70\% de la energía proviene de la combustión del carbón. Por consiguiente, si se busca estabilizar las emisiones en el corto plazo será necesario ampliar la inversión en CAC.

Aunque la tecnología básica de CAC está disponible desde los años noventa, por sus elevados costos económicos y energéticos casi no se utiliza en la generación de ener-

23 Ausubel, J. H. (1996). The Liberation of the Environment. [Versión digital] Daedalus, 125 (3) 1-17. Recuperado de: http://phe.rockefeller.edu/Daedalus/Liberation/. 
gía. La captura purificación y compresión de $\mathrm{CO}_{2}$ es un proceso costoso ${ }^{24}$ por lo que la mayoría de los proyectos en operación forman parte de otros procesos industriales como la producción de gas natural. Aunque la agencia internacional de la energía anticipa que para el 2050 las reducciones de carbono por CAC serán de alrededor del 14\%, el panorama es incierto. Mientras que los críticos señalan que es necesario abandonar el uso del carbón rápidamente, los defensores aseguran que los países en desarrollo utilizarán los combustibles de menor costo. Luego la CAC podría jugar un papel importante en la reducción de emisiones en las próximas décadas. No obstante, su aplicación a gran escala requerirá de grandes inversiones en investigación y desarrollo en el futuro.

La energía solar y la eólica son

muy prometedoras pero todavía

presentan dos limitaciones: su

distribución e intermitencia.

Generalmente los lugares donde

brilla el sol y el viento es fuerte

no coinciden con los centros

urbanos donde se consume la

energía por lo que su utilización

depende de la construcción de

costosas redes de transmisión
Las fuentes de energía renovable constituyen solamente el 13\% de la energía utilizada globalmente. De este valor la biomasa comprende el $79 \%$ y la energía hidroeléctrica el $16 \%$. El $5 \%$ restante lo componen las demás fuentes entre las que se encuentran la energía solar y eólica. En países como Costa Rica y Colombia la energía hidroeléctrica representa una parte importante de la matriz energética, un $80 \%$ y $70 \%$ respectivamente y puede continuar creciendo. Sin embargo en los países que no cuentan con la geografía adecuada la construcción de hidroeléctricas no es viable. Por tanto, la energía hidroeléctrica no puede sustituir a los combustibles fósiles.

La energía solar y la eólica son muy prometedoras pero todavía presentan dos limitaciones: su distribución e intermitencia. Generalmente los lugares donde brilla el sol y el viento es fuerte no coinciden con los centros urbanos donde se consume la energía por lo que su utilización depende de la construcción de costosas redes de transmisión. Esto es importante en áreas continentales de la zona templada donde se consume mucha energía y la irradiación solar y el viento son escasos. Sin embargo, en países afluentes y pequeños como Dinamarca, las nuevas turbinas de viento constituyen una alternativa importante porque el viento es intenso y porque se encuentran conectadas a una matriz regional que incluye plantas de generación hidroeléctricas. Adicionalmente, la energía solar y eólica son intermitentes, lo cual hace que no sean adecuadas para ciudades que requieren energía de forma permanente. Estos dos factores sumados a la dificultad tecnológica para almacenar electricidad y a sus elevados costos hacen que las energías renovables todavía no puedan sustituir a los combustibles fósiles ${ }^{25}$.

Por otra parte, la energía nuclear tiene un costo razonable, puede satisfacer la demanda de grandes ciudades y así reemplazar una fracción importante de los combustibles fósiles. En Francia, por ejemplo, la energía nuclear provee aproximadamente el 75\% de la electricidad. Debido a la inversión estatal sostenida desde los años ochenta, Francia

24 Muradov, N. (2014). Carbon Capture and Storage: In the Quest for Clean Fossil Energy. In Liberating Energy from Carbon: Introduction to Decarbonization. Lecture Notes in Energy (22). New York: Elsevier. Recuperado de: https://doi.org/10.1007/978-1-4939-0545-4_7.

25 Smil, V. (2010). Energy Transitions. Santa Bárbara: Praeger, 190. International Energy Outlook, 2016. www.eia.gov/forecasts/ieo. 
es líder en la construcción de reactores, exporta energía y recibe ganancias considerables $^{26}$. Desafortunadamente la opinión pública no favorece la energía nuclear. En varios países, la ciudadanía se preocupa por la seguridad de los reactores y por el almacenamiento de los desechos. En parte por esta razón, Japón, Alemania y Estados Unidos han reducido la inversión en energía nuclear y están reemplazando algunos reactores nucleares por plantas de carbón lo cual ha aumentado sus emisiones de carbono. Aunque numerosos expertos señalan que los reactores contemporáneos son seguros y que el almacenamiento de los residuos radiactivos se puede resolver, el contexto sociopolítico no favorece la expansión de la energía nuclear.

La transición a fuentes de

energía bajas en carbono es un proceso lento
En suma, el proceso de descarbonización está ocurriendo desde hace más de un siglo impulsado por avances tecnológicos, fuerzas de mercado y por la sustitución gradual en las fuentes primarias de energía. Desde la primera revolución industrial ha ocurrido una transición gradual y parcial desde la madera y el carbón hacia el petróleo y el gas natural. En la próxima década el carbón probablemente continuará impulsando el crecimiento económico en países emergentes, beneficiando a millones de personas, pero causando emisiones considerables.

La transición a fuentes de energía bajas en carbono es un proceso lento. La experiencia histórica indica que el proceso de desarrollo y comercialización de nuevas fuentes de energía obedece a factores tecnológicos y sociopolíticos difíciles de predecir. Aunque una crisis económica o un gran avance tecnológico pueden tener efectos drásticos, es probable que la transición tome varias décadas. Es prudente evitar las simplificaciones y estudiar cuidadosamente la transformación socioeconómica que está ocurriendo en China e India. El aumento constante en la eficiencia y la amplia difusión del gas natural justifican una dosis moderada de optimismo. Por otra parte, el uso de carbón en países emergentes, la baja inversión en energía nuclear en occidente, y las limitaciones de las renovables requieren de más investigación. El futuro de la energía y sus efectos ambientales están por decidirse.

El tercer aspecto del avance tecnológico que complementa el desacoplamiento y la descarbonización es el avance de las tecnologías de la información (TI). La duplicación de la capacidad de procesamiento y almacenamiento de la información ocurre más o menos cada dos años y se debe a un aumento en el número de transistores en un microprocesador. Gracias este proceso, el costo de los ordenadores y del almacenamiento de información se ha reducido notablemente y la capacidad de procesamiento de los teléfonos móviles actuales es millones de veces superior que la de los computadores de los años setenta ${ }^{27}$. En conjunto, los ordenadores, la telefonía móvil y la comunicación a través de internet (plataformas y redes sociales) han revolucionado algunos sectores de la economía. Las sinergias que se generan cuando suficientes usuarios

26 World Nuclear Association (2017). Nuclear Power in France. http://www.world-nuclear.org/information-library/country-profiles/countries-a-f/france.aspx.

Stone R. (2013). Pandora's Promise. New York: Kino Lorber Edu. Recuperado de: http://pandoraspromise.com/.

27 Rifkin, J. (2014). The zero marginal cost society. New York: Palgrave Macmillan. 
tienen a su alcance ordenadores poderosos y una matriz de comunicación instantánea se traduce en beneficios que incluyen desde servicios de transporte y alojamiento compartido, comercio directo, educación gratuita, hasta el diseño virtual de maquinaria sofisticada ${ }^{28}$. Luego no es difícil imaginar cómo la disponibilidad de estas herramientas mejora la eficiencia en uso de los recursos.

Las TI también han potencializado el avance en las ciencias biológicas. Por ejemplo, la agricultura moderna densamente tecnificada, ha permitido mantener constante el área agrícola, aumentar el rendimiento (cosecha) como se mencionó anteriormente y favorecer la recuperación de especies de flora y fauna ${ }^{29}$. De forma similar, la aplicación de la biología molecular ha permitido producir especies vegetales con mejores rendimientos. La mejora sostenida en el uso de la información, en escalas que van desde el gen y el pixel hasta el genoma y la parcela, puede generar importantes mejoras en el manejo de los recursos naturales. Aunque dado que los beneficios y riesgos asociados de estas tecnologías todavía no se conocen bien, es necesario examinarlos de forma cuidadosa sin rechazarlos prematuramente ni adoptarlos de forma pasiva.

\section{Ideas Políticas}

\subsection{Grandes aspiraciones}

\section{Los flujos internacionales de}

capital, la automatización de la

industria y el dinamismo de la

economía global difícilmente

permitirán la creación masiva

de empleo que demandan los

electores y se requiere para

revivir la clase media
Quizás lo más novedoso del ambientalismo moderno es su visión optimista basada en grandes aspiraciones para la humanidad. El manifiesto ecomodernista señala que "un buen antropoceno exige que los humanos empleen sus crecientes poderes sociales, económicos y tecnológicos para mejorar la calidad de vida de las personas, estabilizar el clima y proteger el ambiente". El manifiesto sugiere que el futuro de la humanidad probablemente será mejor que el presente y que si actuamos acertadamente, el futuro también será mejor para otras especies. La apuesta es que es posible tener una sociedad próspera y un medio ambiente sano mediante la construcción de una sociedad tecnológica y políticamente avanzada. Desafortunadamente mientras que las tendencias históricas y los avances científicos sugieren que una transición de este tipo es perfectamente posible, el contexto político es difícil de interpretar.

Modificar el sistema político constituye un desafío que otras sociedades han sabido enfrentar en el pasado. La crisis financiera de 2008-2009 puso en evidencia la fragilidad del sistema económico y la falta de ideas de los líderes políticos. Durante los años siguientes observamos un creciente desempleo y reducciones importantes en la

28 Mason, P. (2016). Postcapitalismo: hacia un nuevo futuro. Barcelona: Paidós.

29 Blomqvist, L. et al. (2015). Nature Unbound: decoupling for conservation. Breakthrough Institute Report. Recuperado de: http://thebreakthrough.org/images/pdfs/Nature_Unbound.pdf. 
inversión social. El impacto social de la crisis permitió que algunos partidos de derecha capturaran el descontento público y pusieran a prueba los valores liberales. Aunque es poco probable que los nuevos proyectos de derecha satisfagan adecuadamente las necesidades de los electores en Europa y Norteamérica, la falta de ideas de la izquierda y del centro liberal es motivo de preocupación. Los flujos internacionales de capital, la automatización de la industria y el dinamismo de la economía global difícilmente permitirán la creación masiva de empleo que demandan los electores y se requiere para revivir la clase media. En consecuencia, garantizar empleo, vivienda, educación y salud para todos los ciudadanos sigue siendo un reto para todos los movimientos políticos. Los gobiernos disponen de escasas herramientas para enfrentar la inestabilidad económica y la desigualdad que caracterizan a la economía globalizada.

Ante este panorama algunos autores afines al ambientalismo moderno señalan que la lucha política, pacífica y democrática es un proceso continuo y que no hay soluciones sencillas para enfrentar la inestabilidad económica. Combatir la desigualdad en mundo donde los intereses de todas las clases sociales están relacionados a través de fondos de pensiones, inversiones bursátiles y bienes raíces, es difícil. Sin embargo, los valores y necesidades de las personas en sociedades postindustriales han sido adecuadamente caracterizados, por lo que podemos desarrollar estrategias para responder a ellos. La siguiente sección describe brevemente los cambios recientes en los valores culturales y su relevancia para movimientos sociales como el ambientalismo moderno.

\subsection{Nuevos valores}

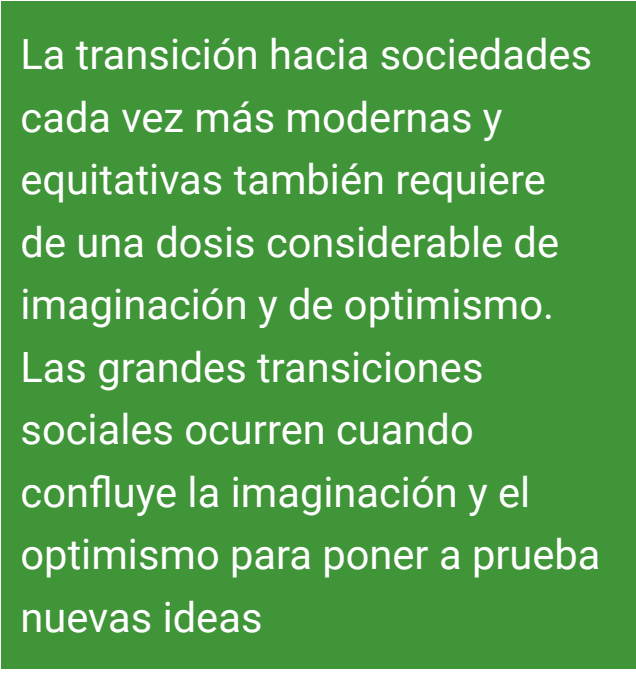

Las sociedades contemporáneas que residen en centros urbanos viven con una mayor afluencia material que hace una o dos generaciones. Para la clase media, la satisfacción de las necesidades básicas es un asunto del pasado. Este mayor nivel de riqueza y el acceso a nuevas tecnologías junto con otros procesos sociales han propiciado cambios profundos en los valores de los jóvenes en las sociedades postindustriales. El reto para los movimientos ambientalistas consistirá en articular adecuadamente estos valores. La experiencia del ambientalismo de tipo alarmista indica que asustar al público y proponer medidas draconianas no funciona. Es necesario construir un movimiento que permita reconciliar intereses que incluyen una mejor calidad de vida, un ambiente sano y un trabajo gratificante en un contexto económico incierto.

Los valores que podrían impulsar los movimientos ambientales en el futuro cercano serán la gratitud y la búsqueda de la excelencia. La gratitud para con los ambientalistas de generaciones anteriores y para con las instituciones apoyan las causas ambientales. La búsqueda de la excelencia debe motivar a la sociedad a tomar riesgos y perseverar en la lucha política animada por la convicción en el potencial de la humanidad para enfrentar los problemas. La transición 
hacia sociedades cada vez más modernas y equitativas también requiere de una dosis considerable de imaginación y de optimismo ${ }^{30}$. Las grandes transiciones sociales ocurren cuando confluye la imaginación y el optimismo para poner a prueba nuevas ideas. Experiencias como el "New Deal" Norteamericano o el crecimiento vertiginoso de China, indican que el cambio social puede ocurrir de forma rápida y sugieren que el futuro está por construir. Por último, el cambio ambiental y social que quisiéramos ver en la próxima generación necesitará inversiones considerables. El avance tecnológico depende de la inversión pública en investigación básica por largos periodos de tiempo. Aunque algunas de las grandes empresas de tecnología de la información parecen no necesitar apoyo del gobierno, la investigación básica en física, biología, medicina, depende todavía en la inversión del estado. Para construir una sociedad tecnológica y políticamente avanzada debemos invertir mayores recursos en investigación básica.

\section{Conclusiones: energía, tecnología y política}

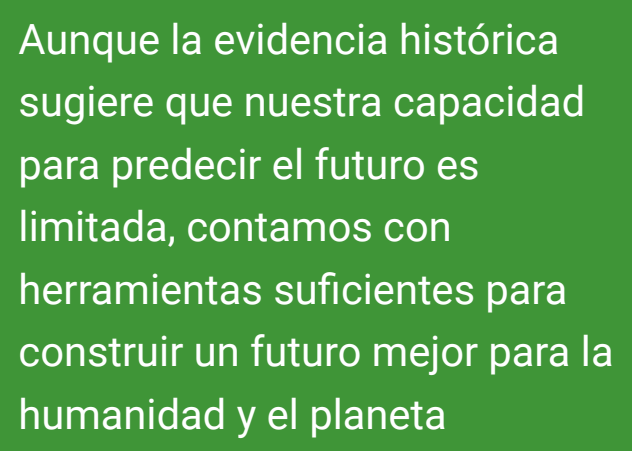

El ambientalismo moderno enlaza eficazmente los objetivos ambientales y sociales alrededor del acceso a la energía. La expansión a gran escala de la matriz energética permitirá cumplir con algunos de los principales objetivos de desarrollo de los países pobres y establecerá las bases para una transición gradual a fuentes de energía más limpias. Este proceso de transición puede retrasarse pero probablemente continuará en las próximas décadas. En segundo lugar, el ambientalismo moderno hace énfasis en la importancia del cambio tecnológico para enfrentar los problemas, por lo que los procesos de desacoplamiento y descarbonización revisten una gran importancia para entender las relaciones entre la economía y el ambiente. Las tendencias históricas indican que los países industrializados están reduciendo sus impactos ambientales gradualmente sin reducir su calidad de vida. Es posible que los países en desarrollo sigan estas trayectorias de forma más acelerada. En tercer lugar, el ambientalismo moderno articula una visión política que resuena con las necesidades de los países en desarrollo y también con algunos desafíos que enfrentan las sociedades postindustriales. La apuesta política se apoya en la convicción en las capacidades humanas para enfrentar los problemas con esfuerzo y creatividad utilizando las herramientas de la ciencia y la tecnología. En este sentido el ambientalismo moderno es cuidadosamente optimista acerca del futuro del ambiente y de la humanidad. Por último, aunque la evidencia histórica sugiere que nuestra capacidad para predecir el futuro es limitada, contamos con herramientas suficientes para construir un futuro mejor para la humanidad y el planeta. 


\section{Agradecimientos}

Las conversaciones con Jenny Price y los comentarios de los revisores anónimos permitieron aclarar muchas de las ideas en el texto.

\section{Referencias}

Asafu-Adjaye, J. et al. (2015). El Manifesto Ecomodernista. http://www.ecomodernism.org/espanol/.

Ausubel, J. H. (1996). The Liberation of the Environment. Daedalus, 125 (3) 1-17. Recuperado de: http://phe. rockefeller.edu/Daedalus/Liberation/.

Ausubel, J. H., Wernick, I. K. and Waggoner, P. E. (2013). Peak Farmland and the Prospect for Land Sparing. [Versión digital] Population and Development Review, 38, 221-242. Recuperado de: https://doi. org/10.1111/j.1728-4457.2013.00561.x.

Blomqvist, L. et al. (2015). Nature Unbound: decoupling for conservation. Breakthrough Institute Report. http:// thebreakthrough.org/images/pdfs/Nature_Unbound.pdf.

Cronon, W. (1983). Changes in the Land: Indians, Colonists, and the Ecology of New England. New York: Hill and Wang.

Dolla, C. N. H. Pachauric, S. (2010). Estimating rural populations without access to electricity in developing countries through night-time light satellite imagery. Energy Policy, 38 (10) 5661-5670.

Fullerton, D. G., Bruce, N., Gordon, S. B. (2008). Indoor air pollution from biomass fuel smoke is a major health concern in the developing world. [Versión digital] Trans R Soc Trop Med Hyg, 102 (9) 843-851. Recuperado de: https://doi.org/10.1016/j.trstmh.2008.05.028.

Kareiva, P., Marvier, M., Lalasz, R. (2012). Conservation in the Anthropocene. Breakthrough Journal. Winter. http://thebreakthrough.org/index.php/journal/past-issues/issue-2/conservation-in-the-anthropocene.

Kareiva, P. (2011). Failed Metaphors and a New Environmentalism for the 21st Century [Video file] https:// www.youtube.com/watch?v=4BOEQkvCook.

Le Quéré, C. et al. (2016). Global Carbon Budget 2016. [Versión digital] Earth Syst. Sci. Data, 8, 605-649. Recuperado de: https://doi.org/10.5194/essd-8-605-2016. http://www.earth-syst-sci-data.net/8/605/2016/. Website: http://www.globalcarbonproject.org/carbonbudget/16/highlights.htm.

Lindeman, T. (2012). 1.3 billion are living in the dark. The Washington Post. Recuperado de: https://www.washingtonpost.com/graphics/world/world-without-power/.

Lomborg, B. (2016). Impact of Current Climate Proposals. [Versión digital] Glob Policy, 7, 109-118. Recuperado de: https://doi.org/10.1111/1758-5899.12295. http://onlinelibrary.wiley.com/doi/10.1111/17585899.12295/full.

Lomborg, B. (2016). The Paris agreement was never the solution to climate change. CAPX. Recuperado de: https://capx.co/paris-was-never-the-solution/ [Video file] https://youtu.be/47bNzLj5E_Q.

Lula da Silva, L. I. (2009). Speech of the Brazilian President Lula at the Conference of the Parties, in Copehagen (COP15), Part 1. [Video file] https://www.youtube.com/watch?v=LQzVjDp5WA8. 
Maniates, M. F. (2001). Individualization: Plant a Tree; Buy a Bike, Save the World? [Versión digital] Global Environmental Politics, 1 (3) 31-52. Recuperado de: https://doi.org/10.1162/152638001316881395.

Mather, A. S. and Needle, C. L. (1998). The forest transition: a theoretical basis. [Versión digital] Area, 30, 117124. Recuperado de: https://doi.org/10.1111/j.1475-4762.1998.tb00055.x.

Mason, P. (2016). Postcapitalismo: hacia un nuevo futuro. Barcelona: Paidós.

Muradov, N. (2014). Carbon Capture and Storage: In the Quest for Clean Fossil Energy. In Liberating Energy from Carbon: Introduction to Decarbonization. Lecture Notes in Energy (22). New York: Elsevier. Recuperado de: https://doi.org/10.1007/978-1-4939-0545-4_7.

Nordhaus T. and Shellenberger, M. (2007). BREAK-THROUGH. New York: Houghton Miflin, 344.

Nordhaus, T. and Shellenberger, M. (2009). Apocalypse Fatigue: Losing the Public on Climate Change. http:// e360.yale.edu/features/apocalypse_fatigue_losing_the_public_on_climate_change.

PEW research center (2009). Fewer Americans See Solid Evidence of Global Warming. http://www.peoplepress.org/2009/10/22/fewer-americans-see-solid-evidence-of-global-warming/.

Rifkin, J. (2014). The zero marginal cost society. New York: Palgrave Macmillan.

Smil, V. (2015). Natural Gas: Fuel for the 21st Century. Wiley, 264.

Smil, V. (2010). Energy Transitions. Santa Bárbara: Praeger, 190.

Speth, J. G. (2005). Red Sky at Morning: America and the Crisis of the Global Environment. New Haven: Yale University Press, p. 328.

Stone R. (2013). Pandora's Promise. New York: Kino Lorber Edu. Recuperado de: http://pandoraspromise.com/.

Swyngedouw, E. (2010). Apocalypse Forever? [Versión digital] Theory, Culture \& Society, 27 (2-3) 213-232. Recuperado de: https://doi.org/10.1177/0263276409358728.

Tierney, J. (2007). 'Feel Good' vs. 'Do Good' on Climate. The New York Times. http://www.nytimes. com/2007/09/11/science/earth/11tiern.html.

United Nations Development Program. Datos sobre el desarrollo humano (1980-2015). Recuperado de: http:// hdr.undp.org/es/data.

Ward, J. D. et al. (2016). Is Decoupling GDP Growth from Environmental Impact Possible? [Versión digital] PLoS ONE 11 (10) e0164733. Recuperado de: https://doi.org/10.1371/journal.pone.0164733.

World Bank. Access to electricity (\% of population) http://data.worldbank.org/indicator.

World Nuclear Association (2017). Nuclear Power in France. Recuperado de: http://www.world-nuclear.org/ information-library/country-profiles/countries-a-f/france.aspx. 